\title{
On the Onset Time of Several SPE/GLE Events: Indications from High-Energy Gamma-Ray and Neutron Measurements by CORONAS-F
}

\author{
Viktoria Kurt, ${ }^{1}$ Karel Kudela, ${ }^{2}$ Boris Yushkov, ${ }^{1}$ and Vladimir Galkin ${ }^{1}$ \\ ${ }^{1}$ Skobeltsyn Institute of Nuclear Physics, Lomonosov Moscow State University, Moscow 119234, Russia \\ ${ }^{2}$ Institute of Experimental Physics, Slovak Academy of Sciences, 04353 Kosice, Slovakia \\ Correspondence should be addressed to Boris Yushkov; clef@srd.sinp.msu.ru
}

Received 5 September 2012; Revised 17 December 2012; Accepted 24 December 2012

Academic Editor: José F. Valdés-Galicia

Copyright (c) 2013 Viktoria Kurt et al. This is an open access article distributed under the Creative Commons Attribution License, which permits unrestricted use, distribution, and reproduction in any medium, provided the original work is properly cited.

\begin{abstract}
We analyzed the high-energy gamma and neutron emissions observed by the SONG instrument onboard the CORONAS-F satellite during August 25, 2001, October 28, 2003, November 4, 2003, and January 20, 2005 solar flares. These flares produced neutrons and/or protons recorded near Earth. The SONG response was consistent with detection of the pion-decay gamma emission and neutrons in these events. We supposed that a time profile of the soft X-ray derivative was a good proxy of time behavior of the flare energy release. Then we showed that time intervals of the maximum both of energy release and pion-decay-emission coincided well. We determined the onset time of GLEs 65, 69 on the basis of neutron monitor data using the superposed epoch method. The time of high-energy proton onset on November 4, 2003 was found from the GOES data. The time delay between the high-energy gamma ray observation and the high-energy protons onset time was $<5$ minutes. This time lag corresponds to the least possible proton propagation time. So, we conclude that in these events both protons interacted in the solar atmosphere and the first protons which arrived to Earth, belonged to one and the same population of the accelerated particles.
\end{abstract}

\section{Introduction}

Solar energetic protons, as Solar Proton Events (SPE) or Ground Level Enhancement (GLE), are observed directly over long time, most probably since the events on February 28 and March 7 in 1942 were identified by Forbush [1] and named later as GLE 1 and 2, respectively. Altogether during the systematic investigation of the GLEs 71 events were recorded. Lower energy of particles, detected by high-latitude neutrons monitors (NM), is $\sim 450 \mathrm{MeV}$ (this threshold is determined by atmospheric absorption), but the effective energy exceeds $1 \mathrm{GeV}$. The minimum energy for medium and low-latitude NM is even higher; it is determined by the geomagnetic cutoff. Satellite measurements allowed us to study accelerated particles below the atmospheric threshold $(\sim 400 \mathrm{MeV})$. A list of SPE events $\left(N(>10 \mathrm{MeV})>10\right.$ protons $\left.\mathrm{cm}^{-2} \mathrm{~s}^{-1} \mathrm{sr}^{-1}\right)$ beginning from 1976 can be found, for example, at [2], http://umbra.nascom.nasa.gov/SEP/, and of GLE events at http://neutronm.bartol.udel.edu/ pyle/GLE_List.txt.
GLE connection with solar flares is not in doubt, but still debated question is whether the protons are accelerated up to subrelativistic energies directly during flare energy release or acceleration occurs later when the shock waves propagate in the upper corona. Direct study of the GLE dynamics does not answer this question, because particles' propagation in the interplanetary magnetic field (IMF) is a complex process controlled by a variety of factors. The onset time depends in particular on particle mean free path, angular separation between the site of observation and the Sun (e.g., [3]). In addition, the magnetospheric transmissivity has to be included to interpret the measurements correctly when observations are done within magnetosphere or on the ground.

However, there are two other channels which allow one to study the scenario of ion acceleration. During solar flares accelerated ions due to interaction with solar atmosphere are producing secondaries, among them neutrals (gamma-rays and neutrons); part of them can also be observed at the Earth's orbit. The advantage of these observations is that there 
is no need of any assumptions on particle propagation in the interplanetary medium and the trajectory change in the magnetosphere. Protons are accelerated to $>300 \mathrm{MeV}$ in the lower corona or they get there after being accelerated in the shock front. They interact with the matter and produce pions which in their turn decay and generate high-energy gammarays with specific spectrum $[4,5]$. The cross-section of pion production increases rapidly to about $1 \mathrm{GeV}$ and then continues to increase slowly. Thus, the high-energy gammarays are generated by protons with energies typical for GLE. Neutrons with energies accessible for detection with NMs can be only produced in the same inelastic interactions of highenergy protons.

The first solar neutron signal at the Earth's orbit was detected by Chupp et al. [6] using measurements with Gamma-Ray Spectrometer (GRS) on Solar Maximum Mission (SMM) during the solar flare on June 21, 1980. Secondtime GRS recorded a solar neutron signal after the June 3, 1982, flare (X8.0/2B, E72S09) [7]. The solar neutron response was observed for the first time at the surface of Earth also in this event by neutron monitor (NM) measurements at Jungfraujoch with $1 \mathrm{~min}$ time resolution [8]. The air thickness along the line of sight to the Sun was $745 \mathrm{~g} \mathrm{~cm}^{-2}$ for the event at Jungfraujoch. Another high mountain NM located in central Europe, namely, Lomnicky Stit $\left(883 \mathrm{~g} \mathrm{~cm}^{-2}\right)$, observed in 5 min records an $\sim 3 \%$ increase in 11:45-11:50 (all times hereinafter are UT). This increase was consistent with characteristic rigidity of accelerated particles 200-250 MV [9]. The energetic protons observed in interplanetary space from this eastern flare were interpreted as the decay products of neutrons. This event was discussed by Evenson et al. [10, 11] and the spectrum of neutrons was obtained in the range 10$100 \mathrm{MeV}$.

The June 3, 1982, flare motivated many neutron monitors to make measurements with higher statistical accuracy and better temporal resolution. It has also demanded the construction of new ground-based detectors (e.g., [12]) and new observation of gamma high-energy emission onboard the satellites. However, the neutron fluxes change significantly as a result of the velocity dispersion and decay of neutrons on the way from the Sun to the Earth (the half-life time of free neutrons is $10^{3} \mathrm{~s}$, which is comparable to the transit time of $1 \mathrm{AU}$ for neutrons with energies $100-1000 \mathrm{MeV}$ ).

There are two "windows" in the electromagnetic emission spectrum which allow us to observe an appearance of accelerated protons in the solar atmosphere. Protons accelerated up to $10-50 \mathrm{MeV}$ excite nuclei in the ambient solar atmosphere. These excited nuclei immediately emit narrow gamma-lines in the energy range of $2-7 \mathrm{MeV}[4,13]$. The most intense lines are at $4.4 \mathrm{MeV}\left(\mathrm{C}^{12 *}\right)$ and $6.1 \mathrm{MeV}\left(\mathrm{O}^{16 *}\right)$ as well as the delayed neutron-capture line at $2.2 \mathrm{MeV}$. It seems that Hirasima et al. [14] were the first to observe solar gammaray lines accompanying the solar flares. Then these lines were also observed by Orbiting Solar Observatory (OSO-7) [15].

A second "spectral window" appears only when the energy of the accelerated protons reached $300 \mathrm{MeV}$. At such energies proton interactions with a matter of the solar atmosphere create neutral pions which decay immediately and generate a broad gamma-ray line with a maximum near $70 \mathrm{MeV}[4,5]$. Charged pions are also produced in the same interactions, and their decay produces electrons and positrons, which, in turn, generate high-energy bremsstrahlung with a broad energy spectrum extending up to the energies of the electrons and positrons themselves. The pion-decay gamma-ray spectrum could ride on top of the continuum deriving from primary electron bremsstrahlung.

Thus, observations of the pion-decay emission during a solar flare provide doubtless evidence of the proton acceleration up to high energies, and of the interaction with dense medium. No other process can produce a spectral feature similar to the pion-decay emission. When high-energy protons interact with a matter, pion-decay gamma-rays are emitted almost instantaneously. So the temporal behavior of the pion-decay emission follows the behavior of the acceleration mechanism intensity. Eliciting pion-decay component in the flare successive spectra allows us to determine with high accuracy the appearance of high-energy protons in the solar atmosphere.

Thus, in the hands of researchers, there are three tools to study processes of proton acceleration to relativistic energies-investigations of GLEs themselves, observations of high-energy gamma-rays, and observations of high-energy neutrons.

The first flare with convincing evidence of pion production was observed with GRS on June 3, 1982, as well as the neutron production $[8,16,17]$. More recently, the pion-decay emission was detected by SMM/GRS [18, 19], GRANAT/Phebus [20-22], Compton Gamma-Ray Observatory (CGRO) with Energetic Gamma-Ray Experiment Telescope (EGRET) [23, 24], and GAMMA/GAMMA1 [25, 26]. This emission was also detected during the declining phase of the solar cycle 23 with the Solar Neutrons and Gamma-rays (SONG) spectrometer [27] on the Complex Orbital Observations of the Active Sun (CORONAS-F) space mission from four solar flares $[28,29]$. A brief review of these observations can be found in $[30,31]$. The latest pion-decay emission observation in two flares during current solar cycle was performed onboard the Fermi mission with Large Area Telescope (LAT) [32]. The reviews of energetic ion properties in the solar atmosphere deduced from observations of neutrons and gamma-rays can be found in $[33,34]$.

The SONG spectrometer on CORONAS-F space mission was working from July 2001 until February 2005 and has increased considerably a poor statistic of the solar events with the data on the pion-decay gamma emission and neutrons. Our team highlighted the pion-decay component during the impulsive phase of August 25, 2001, October 28, 2003, November 4, 2003, and January 20, 2005, powerful flares as well as appearance of neutron fluxes after the first three events.

This work is to some extent a review of our results. Here, after short description of the SONG instrument we discuss the observations of gamma-rays and neutrons during abovementioned events. These observations indicated the presence of protons accelerated up to high energy in solar atmosphere. The association of SPEs and GLEs with these flares is considered. 


\section{CORONAS-F/SONG Data: Analysis of Observations and Method of Spectra Restoration}

A CsI crystal with a diameter of $20 \mathrm{~cm}$ and a height of $10 \mathrm{~cm}$ is the main detecting element of the SONG instrument [27]. The crystal was surrounded by an anticoincidence plastic scintillator shield to reject signals from charged particles. Neutrons with energies $>20 \mathrm{MeV}$ produce recoil protons and other heavy nonrelativistic particles in a CsI(Tl) crystal, whereas gamma photons produce relativistic electrons. The intensity ratio of the fast and slow components of the scintillation pulses in the $\mathrm{CsI}(\mathrm{Tl})$ crystal depends on the particle ionising power, which in turn is different for relativistic electrons and slow heavy particles. This allows us to make discrimination between neutrons and gamma photons. Secondary particles produced by neutrons have a wide energy distribution below the energy of incident neutron. Therefore, the energy of a neutron cannot be determined from its energy deposition.

The time resolution of the SONG detector was 1 or $4 \mathrm{~s}$ depending on the observation mode. In-flight observation conditions and background subtracting procedure are presented in [35].

We studied the flare gamma-ray emission using the response function of the detector simulated with the help of the GEANT 3.21 program. To restore the incident gamma-ray spectrum, we used a two-component model:

(i) a continuum component caused mainly by the primary electron bremsstrahlung;

(ii) a pion-decay component in the form of a broad "line" due to neutral pion decay, peaking at $67 \mathrm{MeV}$ [5], plus a continuum due to bremsstrahlung from electrons and positrons produced in charged pion decay (Murphy, 2009 private communication to VGK).

The continuum components were represented by a power law with gradual steepening at high energies. The latter function was applied in two forms: as a power law with an index gradually changing with energy or as an exponential highenergy cutoff (see more detailed description of the spectra restoration in $[35,36])$. The fitting procedure allows us to obtain both a shape of the continuum spectrum and intensity of the pion-decay component.

We note that it is easier to elicit the pion-decay emission of the flare's spectrum if maximum energy of primary accelerated electrons does not exceed $30-40 \mathrm{MeV}$. If a large number of electrons were accelerated to higher energies, it is sometimes difficult to select small fluxes created by piondecay emission. In these cases, we can specify only an upper limit of the pion-decay component fluxes.

Let us consider four particular events in more detail. In all cases, the total time of flares' observation in our experiment did not exceed $10-15 \mathrm{~min}$. This limit is caused by the peculiarities of the satellite orbit with an altitude of $\sim 500 \mathrm{~km}$ and polar inclination. Observations of flare that generated gamma emission by SONG were restricted due to contamination of the detector count rate by high-energy particles during a passage of CORONAS-F through the radiation belts or due to arriving of solar high-energy particles.
To compare the time behavior of energy release manifested by hard X-ray emission and derivative of soft X-ray emission with time profile of the pion-decay emission, we used, in addition to SONG data, the Solar Spectropolarimeter (SPR-N) [37] data and GOES soft X-ray measurements. SPR$\mathrm{N}$ is a monitor of hard X-ray emission. It had two energy bands of 15-40 and of 40-100 keV and operated also onboard the CORONAS-F. It has never been saturated during the events under consideration.

\section{SONG Observations during the Solar Flares}

3.1. Solar Flare on August 25, 2001. The impulsive phase of the August 25, 2001, solar flare (GOES X5.3/3B, S17E34) was characterized by a gradual increase in the total intensity and energy of accelerated particles (see [38-41]). It lasted in highenergy emission band not more than 4-5 min. Time profiles of soft X-rays, the soft X-ray derivative, $d I_{\mathrm{SXR}} / d t$, hard X-ray, and gamma emissions observed during the flare are presented in Figure 1. The background in the CORONAS-F data has been subtracted. The time profile of $d I_{\mathrm{SXR}} / d t$ normalized hereinafter to its maximum value shows that maximum energy input into emitting volume took place near 16:31:30. This time moment corresponds to maximum intensity of hard X-ray (40-100 keV) and gamma-ray (>60 MeV) emissions. Peak time of gamma emission with energies $0.3-2 \mathrm{MeV}$ is slightly delayed. This time difference of hard X-rays with various energies is a consequence of the magnetic trapping of electrons near the loop top and the subsequent precipitation into the footpoint. Unlike the no-diffusion case, electrons can escape from the magnetic trap via pitch-angle scattering which is strongly energy dependent. A detailed discussion of this issue is out of scope of this work.

The incident energy spectra of gamma-ray emission accumulated in three time intervals are shown in Figure 2. These spectra were calculated with fitted parameters of the incident spectra in the two-component model discussed above. Figure 2 demonstrates changing in the spectrum shape and intensity of the pion-decay emission which dominates at energies above $60 \mathrm{MeV}$. Maximum of pion-decay emission was at 16:31:38-16:31:46, that is, close to the peak time of $d I_{\text {SXR }} / d t$ and hard X-rays with energy of $40-100 \mathrm{keV}$. The time interval of energy release maximum can be defined hereinafter as an interval when $d I_{\text {SXR }} / d t$ value exceeds 0.9 of its maximum. Thus, time intervals of maximum energy release and of maximum pion-decay emission overlap.

We got an opportunity to build a combined spectrum using GOES soft X-ray, SPR-N, SONG, and Yohkoh (Hard $\mathrm{X}$-ray Spectrometer (HXS) and Gamma-Ray Spectrometer (GRS) [42]) data. Figure 3 shows this spectrum, accumulated over 16:31:38-16:31:46. To restore SPR-N and SONG spectrum we used an algorithm described in [43]; spectra of Yohkoh/HXS and Yohkoh/GRS were restored by Kashapova (private communication). This spectrum demonstrates that the primary electron continuum with power law lasts up to 60-100 MeV. The narrow gamma-lines and the line of piondecay were identified over it. The flare also provided clear signals of neutron production with sufficient intensity to be recorded by CORONAS-F/SONG [28] after 16:35 and by the 


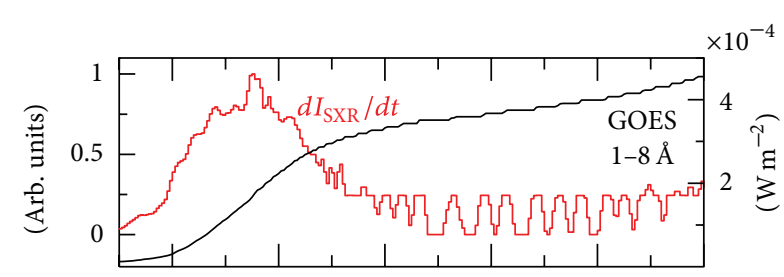

(a)

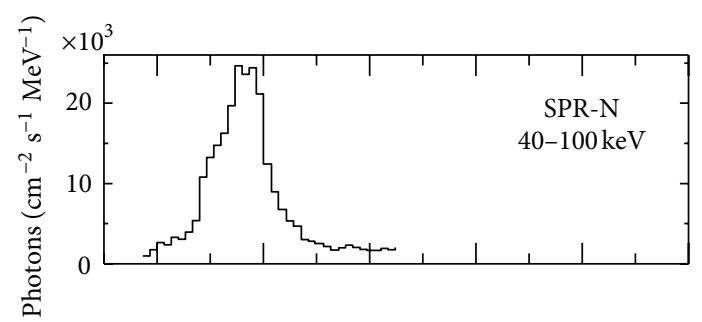

(b)

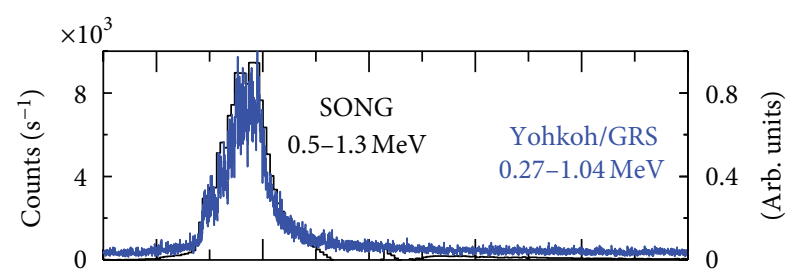

(c)

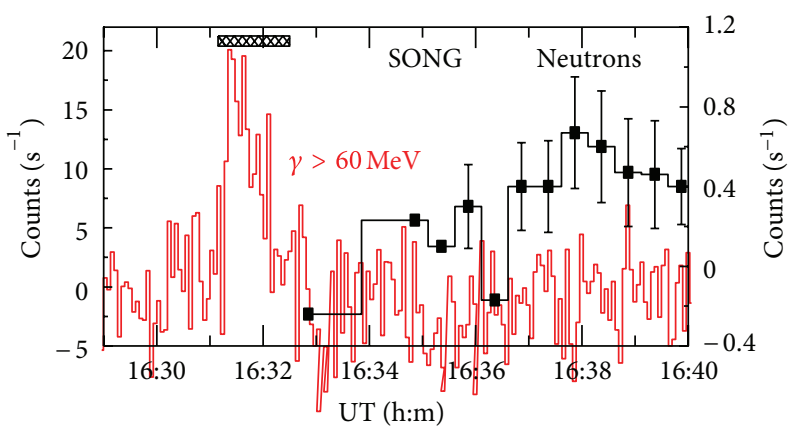

(d)

Figure 1: Time profiles of selected emissions from the August 25, 2001, flare: (a) soft X-ray flux (hereinafter black curve and right $y$-axis) and its derivative (hereinafter red curve and left $y$-axis); (b) hard X-ray $40-100 \mathrm{keV}$ from CORONAS-F/SPR-N; (c) gamma-rays $0.5-1.3 \mathrm{MeV}$ (CORONAS-F/SONG, black curve, left $y$-axis) and $0.27-1.04 \mathrm{MeV}$ (Yohkoh/GRS, blue curve, right $y$-axis; http://solar.physics.montana.edu/sxt/); (d) gamma-rays $>60 \mathrm{MeV}$ (red, left $y$-axis) and neutrons (black, right $y$-axis) from CORONAS-F/SONG. Shaded rectangle in bottom panel denotes hereinafter the interval of undoubted observation of the pion-decay gamma-emission.

Chacaltaya NM in 16:35-16:36 [44]. This time lag relative to $16: 31: 30$ corresponded to neutron energies $>300 \mathrm{MeV}$. No GLE was observed after this eastern event.

3.2. Solar Flare on October 28, 2003. This prominent flare (X17.2/4B, S16E08) was observed in the soft X-ray and in

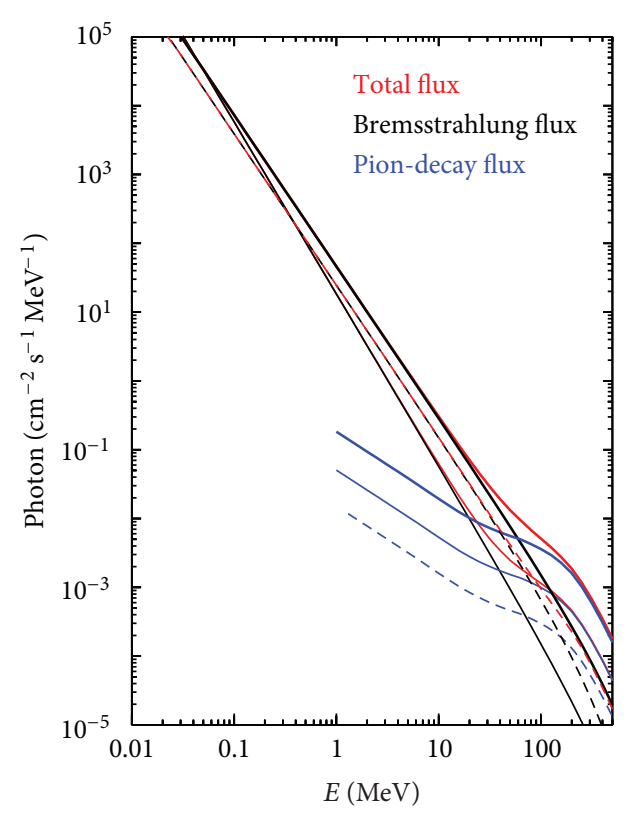

FIgURE 2: Restored energy spectra of gamma-rays for different time intervals. Thin lines correspond to 16:31:02-16:31:14, thick lines to 16:31:38-16:31:46, and dashed lines to 16:32:02-16:32:10. Red curves represent the total spectra, black curves electron bremsstrahlung, and blue curves the pion-decay component.

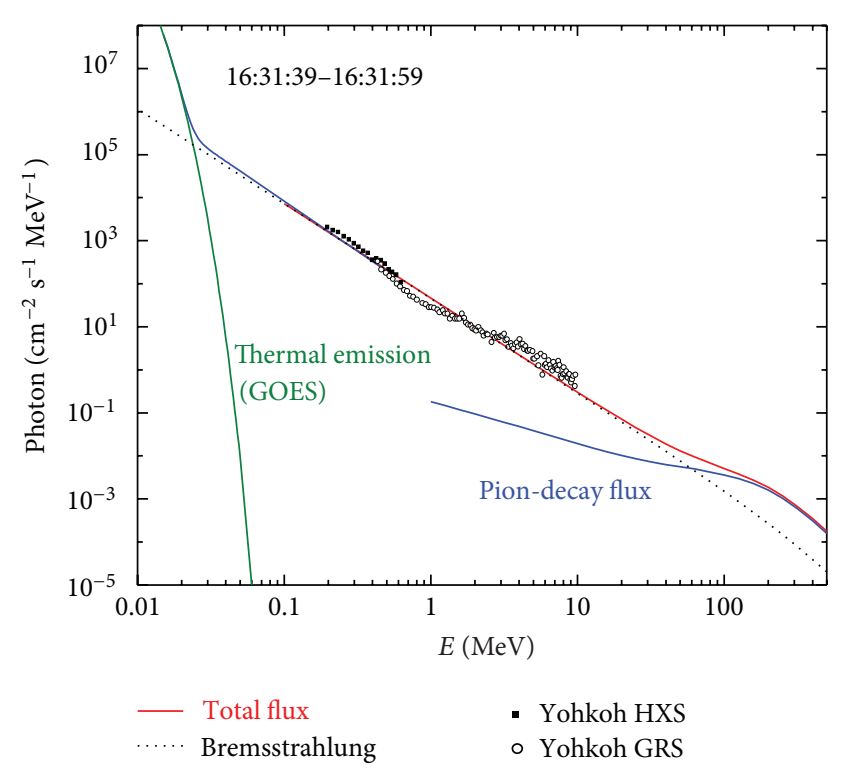

Figure 3: Combined spectrum of the August 25, 2001, flare. The total flux (blue curve) was restored from CORONAS-F data, the piondecay flux-from SONG data.

H $\alpha$ ranging from 09:40 to 18:00 (http://spidr.ngdc.noaa.gov/ spidr/). According to measurements within various ranges, the beginning of the impulsive phase (the beginning of the main energy release of this flare) was determined as 11:01-11:02 (see, e.g., [45-47]).

The increase in the hard X-ray emission over the background level caused by the solar flare was reliably detected 


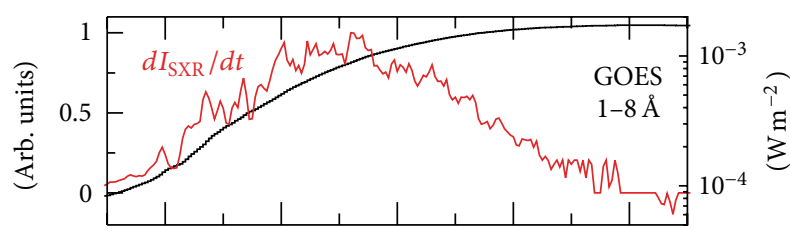

(a)

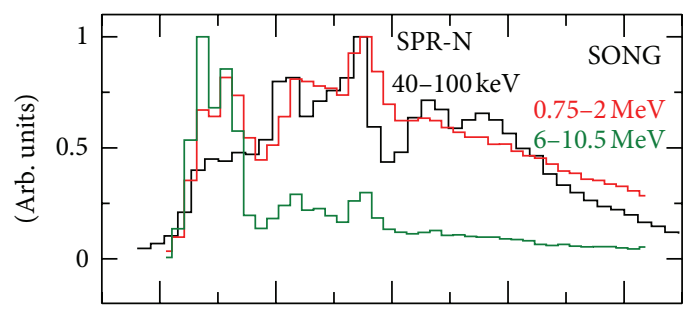

(b)

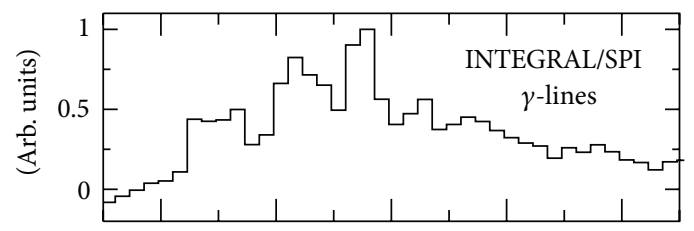

(c)

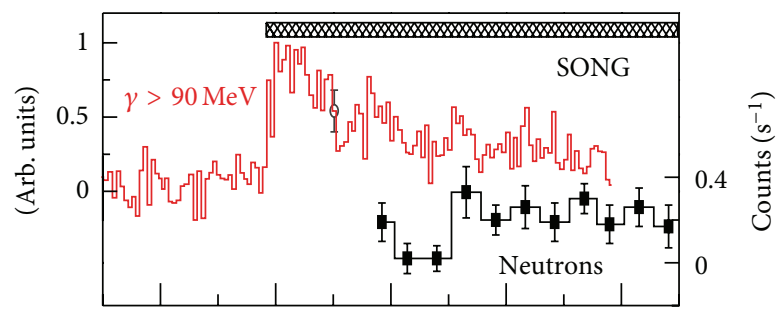

(d)

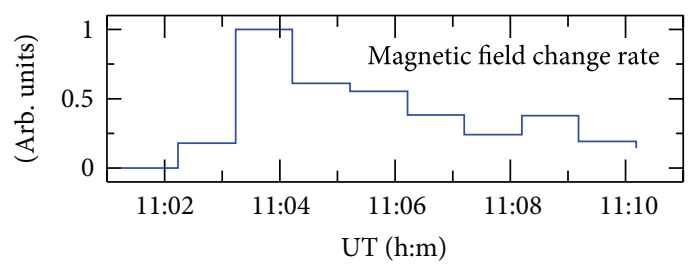

(e)

FIGURE 4: Normalized time profiles of selected emissions from October 28, 2003, flare: (a) soft X-ray flux and its derivative; (b) hard X-ray 40-100 keV from CORONAS-F/SPR-N (black curve); gamma-rays from CORONAS-F/SONG 0.75-2 MeV (red) and 6$10.5 \mathrm{MeV}$ (green); (c) gamma-lines from INTEGRAL/SPI [46]; (d) gamma-rays $>90 \mathrm{MeV}$ (red, left $y$-axis) and neutrons (black, right $y$-axis) from CORONAS-F/SONG; (e) magnetic field change rate [48].

by the SONG instrument beginning from 11:02:11 to 11:12:30 (see Figure 4). This Figure presents also summarized intensity of the $4.4+6.1 \mathrm{MeV}$ gamma-lines (INTEGRAL with the SPI spectrometer [46]) and the magnetic field change rate obtained with $\sim 1$ min cadence [48].

The detailed study of SONG hard X-ray and gammaray emission measurement of this flare and neutrons can be
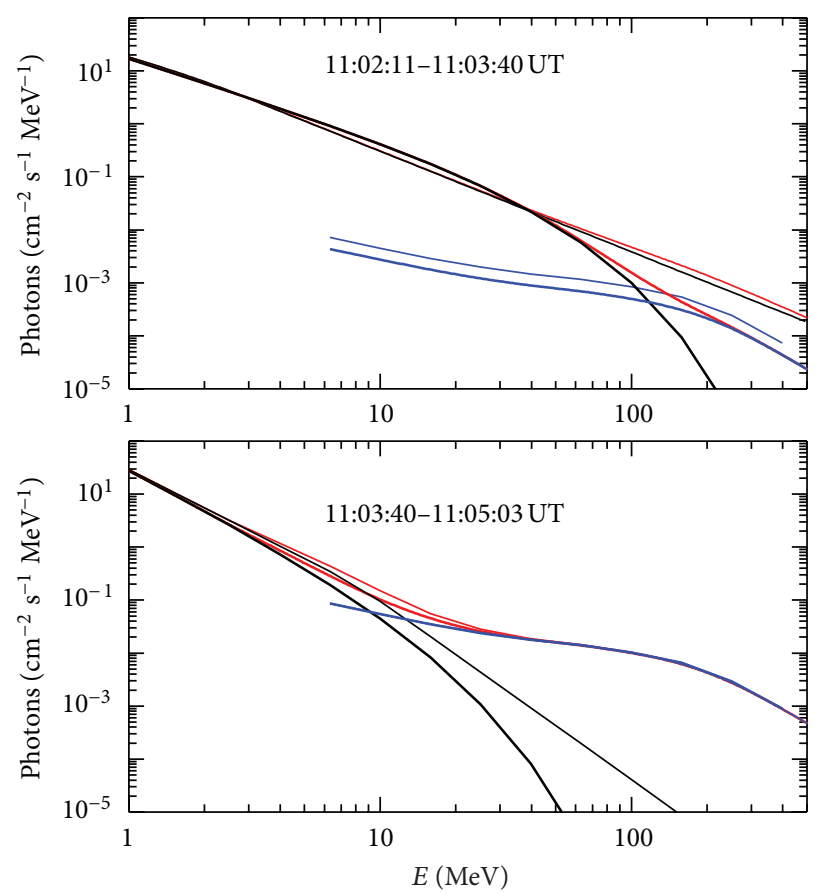

FIGURE 5: The restored incident gamma-ray spectra of the flare on October 28, 2003 (from [35]). Red curves represent the total spectra, black curves electron bremsstrahlung component, and blue curves the pion-decay gamma-ray spectrum. The thin curves correspond to a power law with varying index and the thick curves correspond to a power law with an exponential cutoff.

found in [35]. The important interval is 11:02:21-11:03:40, when power law spectrum caused by the electron bremsstrahlung extended up to very high energies of photons $(100 \mathrm{MeV})$ was observed. We could not distinguish piondecay contribution in this spectrum; consequently we could estimate the upper limits of pion-decay flux if any. At 11:03:51 a sharp increase of gamma-ray emission with energies above $40 \mathrm{MeV}$ occurred. The shape of the spectrum changed substantially in this moment: a characteristic feature has appeared at energies above $30 \mathrm{MeV}$ caused by the decay of neutral pions (see Figure 5). It was very surprising that the continuum created by primary electrons became more soft with a break around $40 \mathrm{MeV}$ during this second energy release episode.

Comparing narrow gamma-line intensities [46] with the pion-decay flux at $100 \mathrm{MeV}$ energy Kuznetsov et al. [35] has showed that the spectrum shape of the accelerated protons had also changed from exponential (11:02:21-11:03:40) to power law with spectral index equal to $\sim 3$ after 11:03:51. This spectrum shape remained virtually unchanged, whereas the total intensity was decreasing gradually till 11:12, which was the end of our measurements. Regarding the time profile of hard X-rays and $d I_{\mathrm{SXR}} / d t$ it is evident that both curves reach their maximum values at 11:05-11:06 simultaneously with the time interval of maximum intensity of the piondecay emission.

High-energy neutrons were measured by the SONG detector beginning at 11:06:20 $[28,35]$ and by the Tsumeb 


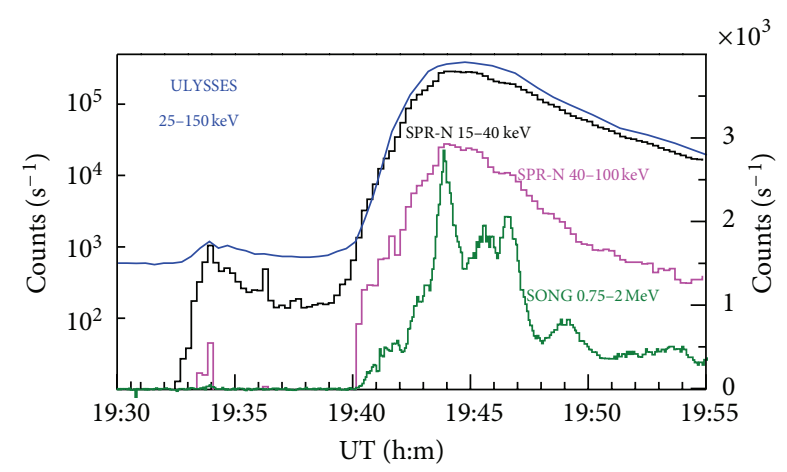

FIgURE 6: Time profiles of selected emissions from the November 4, 2003, flare measured by Ulysses and CORONAS-F. Left $y$-axis is suitable to Ulysses and SPR-N (15-40 keV) data; right $y$-axis to SPR$\mathrm{N}(40-100 \mathrm{keV})$ and SONG data.

NM [49]. Bieber et al. [49] estimated the onset time of the neutron generation on the Sun to be 10:56:30 $\pm 1 \mathrm{~min}$, which corresponded to the time of the significant increase in the pion-decay emission (11:03:50-500 s) observed by the SONG detector.

3.3. Solar Flare on November 4, 2003. A new possibility to study the behavior of high-energy gamma-rays appeared during an unusually powerful flare on November 4, 2003 (X > 28/4B, S19W83), which occurred at the active region NOAA 10486 that approached the limb. The soft X-ray detectors onboard the GOES satellites were saturated within the 19:4319:58 interval. However, soft X-ray importance $>$ X28 was later attributed to this flare. In addition to a very powerful soft $\mathrm{X}$-ray burst, radio emission up to $405 \mathrm{GHz}$ frequency was observed [50].

CORONAS-F satellite came out from the radiation belt region at 19:42:14 and was observing high-energy gammarays from this flare till the arrival of the intense flux of solar neutrons at 19:48:00-19:48:30. Beginning from this moment we could not reliably separate the detector signals with energies $>20 \mathrm{MeV}$ caused by photons from the signals caused by neutrons.

Since the flare was partly a behind-the-limb one, a question arises: do our measurements cover a time interval of the flare main energy release or they do not? We compared our data with the measurements of the $25-150 \mathrm{keV}$ hard X-ray emission by the Ulysses spacecraft located at $\sim 114^{\circ}$ westward from the Earth-Sun line at a distance of 5.28 AU from the Sun and measured X-ray emission from 19:33 [51]. The maximum of the Ulysses count rate was observed at approximately 19:44 (see Figure 6) taking into account the distance to Ulysses.

Figure 6 also shows the count rates of the hard Xray emission measured by the SPR-N and SONG detectors onboard the CORONAS-F. One can see that a small intensity flare preceded the major flare (see also [52]). The main flare energy release onset was at 19:38:30. CORONAS-F started its observation at 19:42:14. This time by a lucky occasion is close to the beginning of the main energy release. Thus, $10 \mathrm{~min}$ of our measurements covered practically the total time interval of the flare impulsive phase except the very beginning of the event between 19:38:30 and 19:42:14. Figure 7 shows in more detail the time behavior of various flare emissions.

Spectral analysis revealed a small contribution of the pion-decay component at the level $>1 \cdot 1^{-4}$ photons $\mathrm{cm}^{-2} \mathrm{~s}^{-1} \mathrm{MeV}^{-1}$ within the time interval 19:42:1419:42:40. The maximum of the gamma-rays from the pion decay is equal to $1 \cdot 10^{-3}$ photons $\mathrm{cm}^{-2} \mathrm{~s}^{-1} \mathrm{MeV}^{-1}$ (see Figure 8).

The high-altitude NMs at Mexico and Haleakala [53] and the SONG instrument [28] recorded intense fluxes of solar neutrons whose energy was estimated as $200 \mathrm{MeV}$ on the basis of the time of flight method.

GLE associated with this flare was not observed, though the fluxes of solar protons with energy $>700 \mathrm{MeV}$ were recorded with High-Energy Proton and Alpha Particles Detector (HEPAD) on GOES (http://umbra.nascom.nasa .gov/SEP/).

3.4. Solar Flare on January 20, 2005. Throughout the history of flare studies the solar flare which occurred on January $20,2005,(X 7.1 / 3 B, N 14 W 61)$ is apparently the event with the best set of various experimental data (see, e.g., $[36,54,55]$ ). Radio data [54] and Ramaty High-Energy Solar Spectroscopic Imager (RHESSI) measurements indicated that intense acceleration of electrons up to energies $100 \mathrm{keV}$ and protons up to energies $10-30 \mathrm{MeV}$ began at approximately 06:42. Time profiles of selected emissions are presented in Figure 9. The increase of hard X-rays $(>40 \mathrm{keV})$ and gamma-rays in the SPR-N and SONG data could be distinguished over the background count rates after 06:43:30.

Several distinguished episodes of energy release which led to the change in the emission spectrum shape are clearly seen in Figure 9. The maximum time of $d I_{\mathrm{SXR}} / d t$ coincides well with the maximum time of the energetic emission at 06:47:10 (see panels (c), (d), and (e)). At the same time the intensity of hard X-rays (40-100 and 100-300 keV) falls dawn. This decrease was probably caused by the reverse current, created by a very intense electron beam (see, e.g., $[43,56]$ ). We merely note that accelerated protons can carry an additional input in the cumulative energy budget of the emitting plasma and we do not discuss this experimental fact in more detail, since it is beyond the scope of the present work.

Figure 10 presents four successive emission spectra calculated with various forms of continuum spectrum. Each of them was accumulated over $1 \mathrm{~min}$. The figure demonstrates a gradual increase in the total intensity of gamma-rays. The plateau caused by pion-decay becomes distinctly pronounced over 06:44:40-06:48:40 time interval. This spectral peculiarity was distinguished even within the 06:44-06:45 interval at the very low level of (1-3) $\cdot 10^{-4}$ photons $\mathrm{cm}^{-2} \mathrm{~s}^{-1} \mathrm{MeV}^{-1}$ at the $100 \mathrm{MeV}$ energy. Beginning from 06:45:40, a substantial increase of intensity of photon with energies $>10 \mathrm{MeV}$ is observed. The maximum of the high-energy gammaray emission was observed at 06:46-06:47 and was 3.6 . $10^{-3}$ photons $\mathrm{cm}^{-2} \mathrm{~s}^{-1} \mathrm{MeV}^{-1}$ at the $100 \mathrm{MeV}$. Note that the 


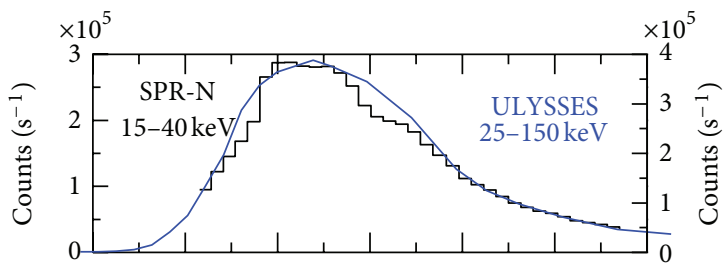

(a)

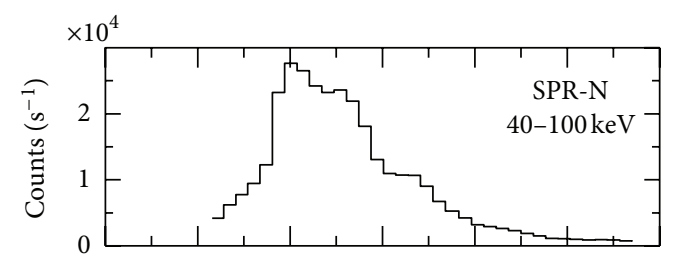

(b)

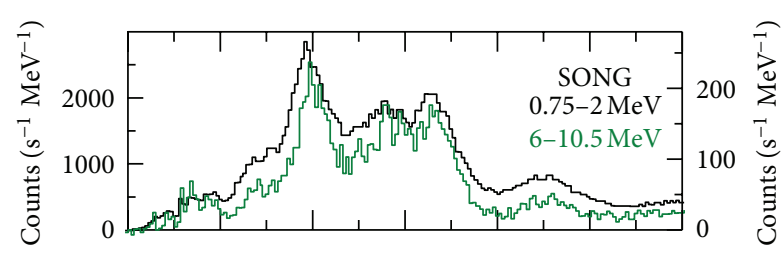

(c)

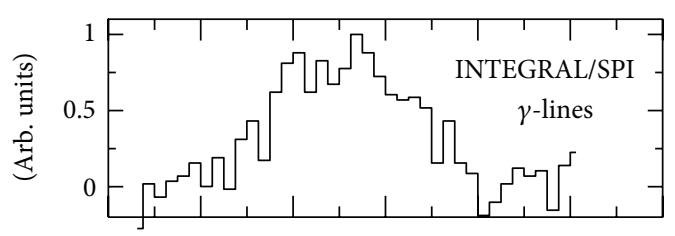

(d)

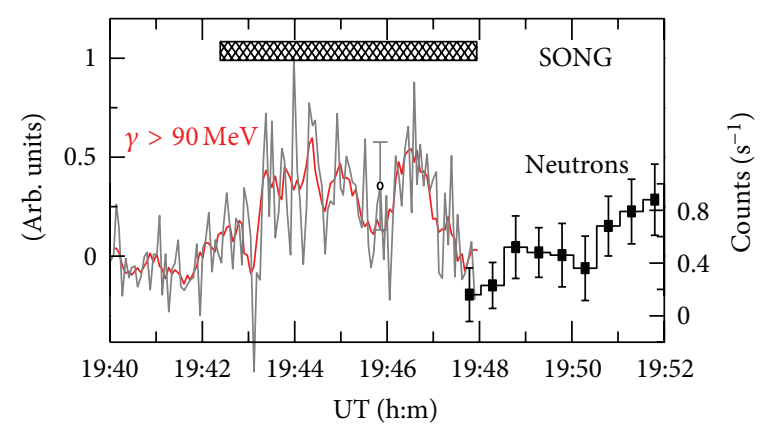

(e)

FIGURE 7: Time profiles of selected emissions from the November 4, 2003, flare: (a) hard X-ray measured by Ulysses (blue curve, right $y$ axis) and CORONAS-F/SPR-N (black curve, left $y$-axis); (b) hard X-rays (40-100 keV) from CORONAS-F/SPR-N; (c) gamma-rays $0.75-2 \mathrm{MeV}$ (black, left $y$-axis) and $6-10.5 \mathrm{MeV}$ (green, right $y$-axis) from CORONAS-F/SONG; (d) summarized normalized intensity of the 4.4 MeV + 6.1 MeV gamma-lines from INTEGRAL/SPI [53]; (e) gamma-rays $>90 \mathrm{MeV}$ (gray, left $y$-axis, data are normalized) and neutrons (black, right $y$-axis) from CORONAS-F/SONG. Red curve in the bottom panel presents smoothed data on gamma-rays.

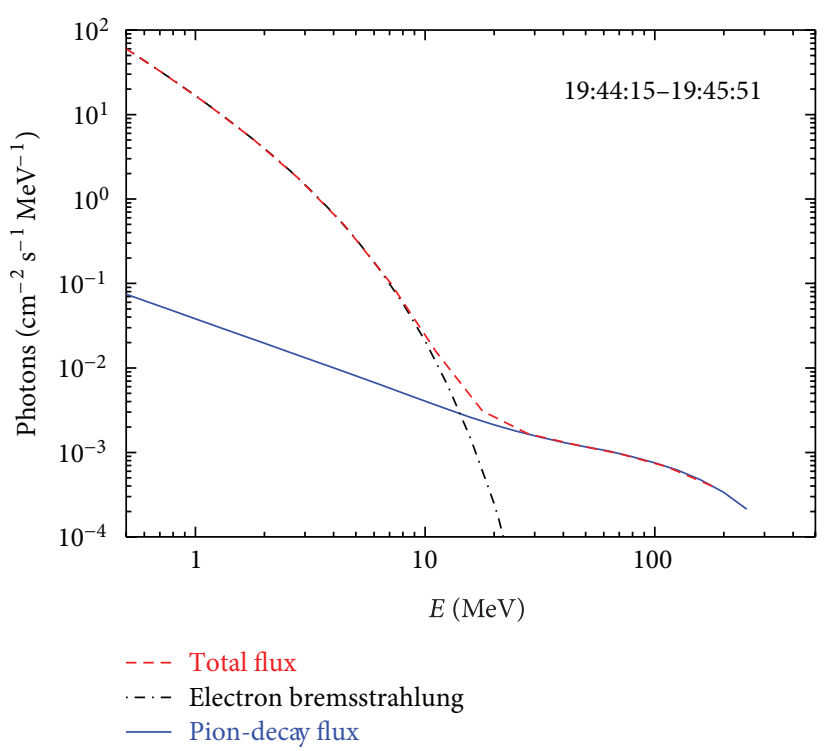

FIGURE 8: The restored incident gamma-ray spectrum of the flare on November 04, 2003. Red curve represents the total spectra, black curve electron bremsstrahlung component, and blue curve the piondecay gamma-ray spectrum.

intensity of the pion-decay emission does not depend appreciably on a choice of continuum spectrum. Maximum pionproduction coincided with time of maximum energy release.

The protons accelerated on the Sun were observed by the world NM network (GLE69) [2].

\section{GLEs and SPE Onset Associated with the Discussed Flares}

The time profile of the pion-decay gamma emission corresponds to the time evolution of the population of the interacting protons and can be used to determine, at least, the onset time of proton acceleration. Further, the most important issue is to know the timing between the particle acceleration up to relativistic energies and time of release of protons, in some cases connected with GLE observations.

Suppose that a certain number of the accelerated protons escaped immediately into space after acceleration. So, if the onset or maximum time of the pion-decay emission was observed, we know the escaping time of the particles. In fact, this assumption means that protons interacting at the Sun (and generating pions) and protons responsible, at least for the initial phase of the GLE, should belong to the same population of accelerated particles.

Usually, when one analyzes the propagation of solar cosmic rays in the interplanetary space the ideal Parker model of the solar wind (SW) is used. It suggests that the IMF lines are in the form of Archimedean spirals. Their parameters (length and heliolongitude of magnetic field line connecting the Sun and Earth) are determined by averaged values over few days prior to the flare. The mean free path of individual particles may be less than the length of the nominal values of 


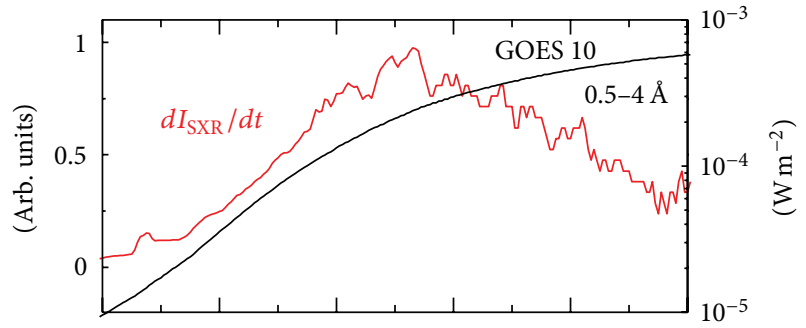

(a)

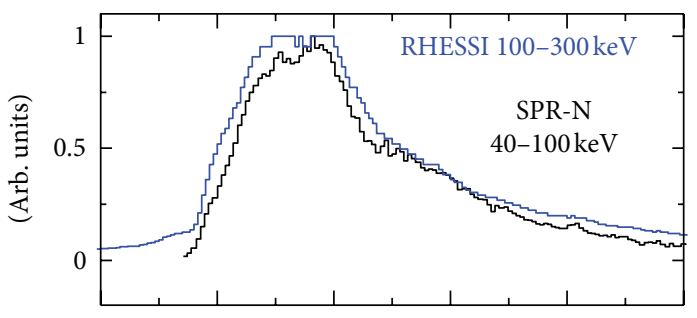

(b)

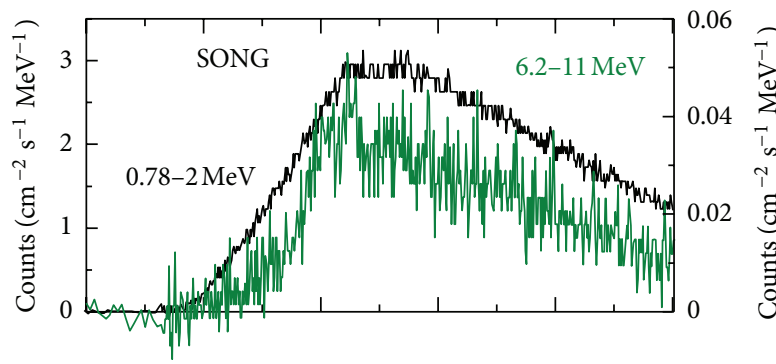

(c)

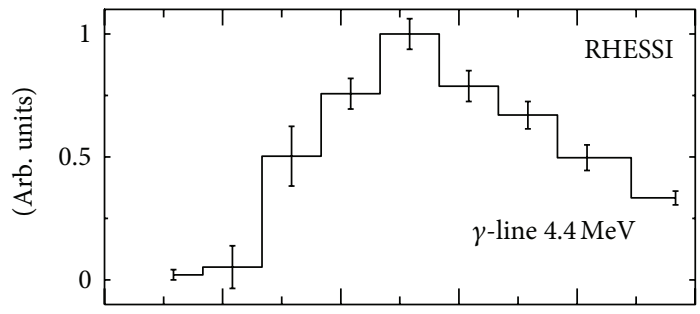

(d)

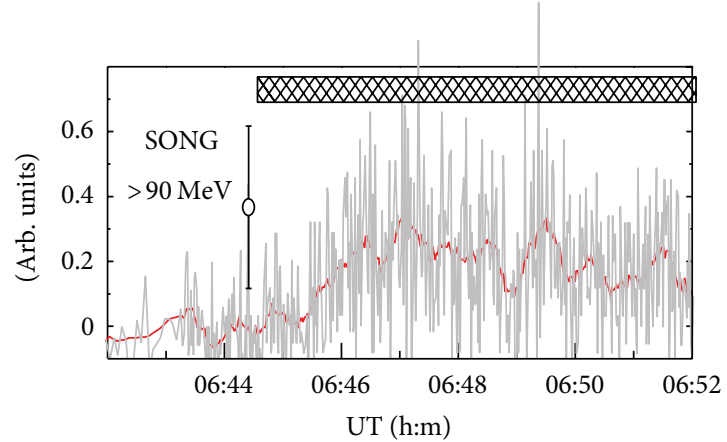

(e)

Figure 9: Time profiles of selected emissions from the January 20, 2005, flare: (a) soft X-ray flux (black curve) and its derivative (red curve); (b) hard X-rays (SPR-N and RHESSI, arbitrary units); (c) gamma-rays $0.78-2 \mathrm{MeV}$ (black, left $y$-axis) and $6.2-11 \mathrm{MeV}$ (green, right $y$-axis) from SONG; (d) the $4.4 \mathrm{MeV}$ gamma line from RHESSI, courtesy G. Share; (e) high-energy gamma-rays (SONG). Red curve in the bottom panel presents smoothed data on gammarays.
IMF line, but not less than the distance from the Sun to Earth in this epoch (see, e.g., [57]).

It should be added that relativistic particle events are observed at times of high solar activity, when transient magnetic structures such as CMEs disrupt often the IMF structure as, for example, in the intervals August 1972, August 1989, June 1991, and October-November 2003. Therefore, in these cases it is unlikely to assume the regularity of the spiral structure of IMF. Sun-Earth magnetic connections can be rooted far from the nominal Parker spiral (see, e.g., $[58,59]$ ).

The time lag between the onset of particles detected on Earth with respect to the particle release time from the Sun can be estimated from the following suggestions.

(i) Let us assume that accelerated particles escaping from the Sun get on the shortest IMF lines with a small pitch angle.

(ii) Particles are propagating with small or without scattering. Therefore, their trajectory lengths are close to the length of the IMF line.

(iii) The threshold energy of the particles detected by high-latitude $\mathrm{NM}$ is $\sim 450 \mathrm{MeV}$ due to an atmospheric absorption, but the effective energy of the particles detected by high-latitude neutron monitors exceeds $1 \mathrm{GeV}$ (see, e.g., [60]). The latter energy corresponds to the particle velocity not less than the $v=0.875 c$ ( $c$ is the speed of light). The energies of particles detected by the medium and low-latitude NMs are even higher, and these energies are determined by the geomagnetic cutoff.

(iv) The propagation time of protons with energy of $1 \mathrm{GeV}$ assuming the path length between 1 and $1.3 \mathrm{AU}$ is equal to $581-755 \mathrm{~s}$, respectively.

(v) If this is true, we expect that a little short impulse or raising intensity springs out before the main event enhancement (i.e., a kind of precursor). It can be observed by one or several NMs with the lag, approximately $670 \pm 80 \mathrm{~s}$ relative to particle release time.

Let us compare the release time of these leader particles with the time of an efficient generation of protons in the flare (as determined by timing of the pion-decay emission). Remember that the propagation time of $1 \mathrm{AU}$ for gamma photons is equal to $\sim 500 \mathrm{~s}$. Thus, if we find that the precursor's particles were delayed with respect to gamma-rays at the $\approx 3-$ $5 \mathrm{~min}$, we get the reason to believe that these protons were accelerated in the flare. Given the spread of path lengths and the uncertainty of data on the velocities of the first recorded particles, their delay relative to the observation time of the pion-decay gamma-rays should fall within the interval of 1-6 minutes.

Let us compare GLE 65 and 69 onset as well as SPE onset of November 4, 2003, with the time of pion-decay emission measurements during associated flares which occurred on October 28, 2003, January 20, 2005, and November 4, 2003.

First of all we determined the beginning of observation of GLEs 65, 69 [61]. We have carefully studied the excess above the background level in the analysis of all available NMs data. 

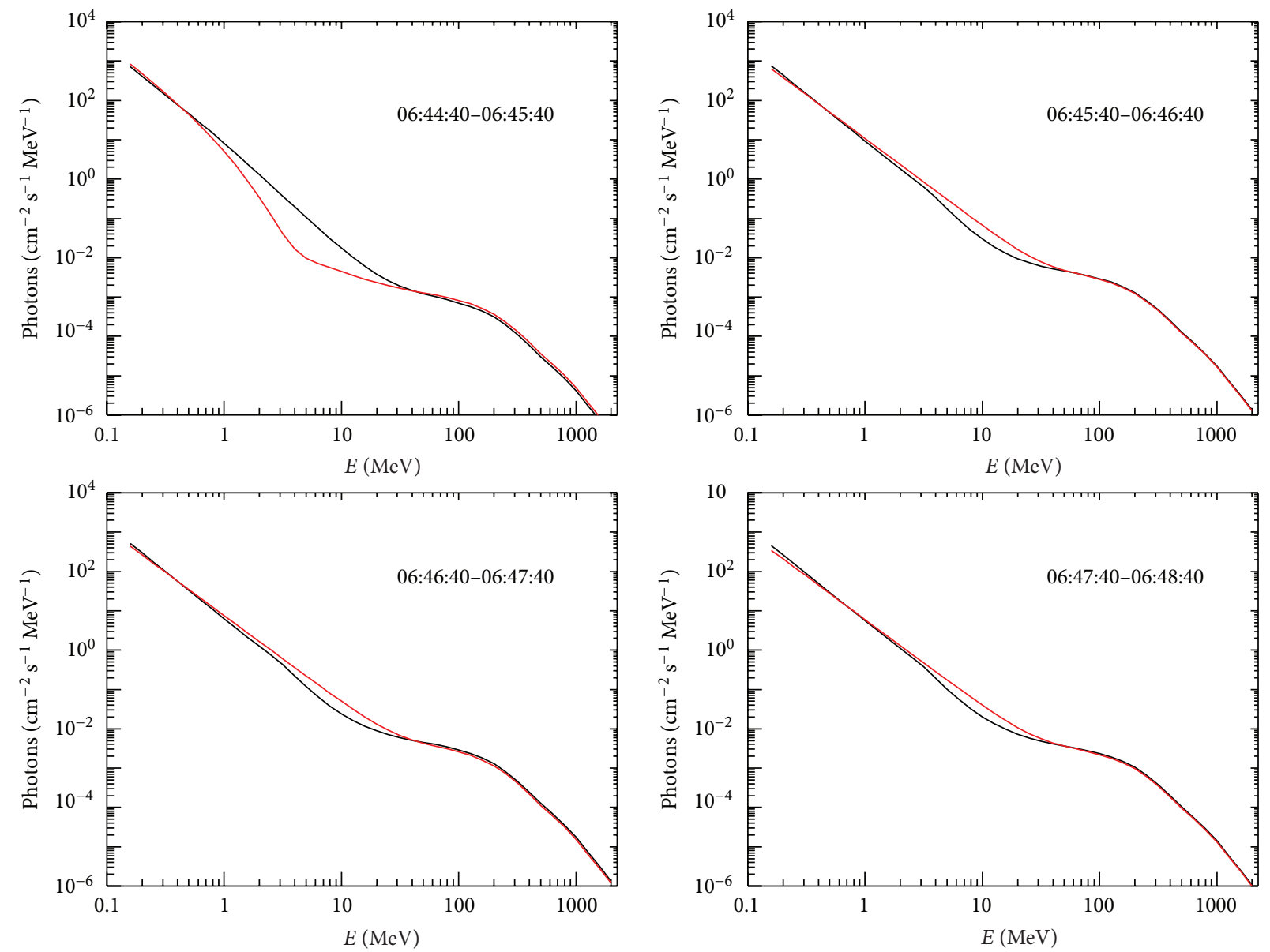

FIGURE 10: The total energy spectra of photons during the flare on January 20, 2005. The red curves correspond to a power law with varying index and the black curves correspond to a power law with an exponential cutoff.

If this excess is seen just in data of a single NM, it may be random, even if its value exceeds $3 \sigma$ level. To determine the interval of first particles arrival, we used the method of superposed epoch for the data from several stations hinting a notional excess.

Figures 11 and 12 illustrate our method and the results obtained in GLE65 and GLE69. For GLE65 data of Hermanus, Irkutsk-2, McMurdo, Cape Shmidt, and Calgary NMs were used. The proton acceleration time and the maximum energy release were 11:04-11:06, and we determined the statistically significant time of the first particles' arrival as 11:06-11:08.

For GLE69 data of Oulu, Norilsk, Apatity NMs were used. In addition we show the NM South Pole data because these data are commonly used to demonstrate the onset of GLE 69 at 06:48-06:49. Our method determined the time of the first particle arrival as 06:47-06:48.

The proton acceleration time and the maximum energy release were 06:46-06:48.

To check the possibility of direct observation of solar neutrons by selected NMs we calculated an angle between the local vertical and the direction to the Sun at the time of the event under consideration [61]. Practically all cases of solar neutron observations were performed by high-altitude
NMs located near the subsolar point (e.g., the Tsumeb NM during the event on October 28, 2003 [49]). NMs whose data were used in this work had not a possibility to observe solar neutrons.

No GLE event associated with the November 4, 2003, flare was reported. We used data of the HEPAD detector onboard GOES-10 and -12 and found the high-energy (>700 MeV) proton onset of the SPE between 19:45 and 19:55 (see Figure 13). The proton acceleration time and the maximum energy release were 19:44-19:48.

In Table 1 we summarize the timing of pion-decay emissions and high-energy particle onsets for events examined in this work.

\section{Discussion}

Before the launch of CORONAS-F data set of the flares with the pion-decay emission consisted only of 8 events. CORONAS-F measurements extended this set essentially. 120 flares with hard X-ray emission were observed with CORONAS-F, and high-energy electron bremsstrahlung with photon energy $>5 \mathrm{MeV}$ was observed in 15 flares. We were lucky and have measured gamma-rays with energies up to 

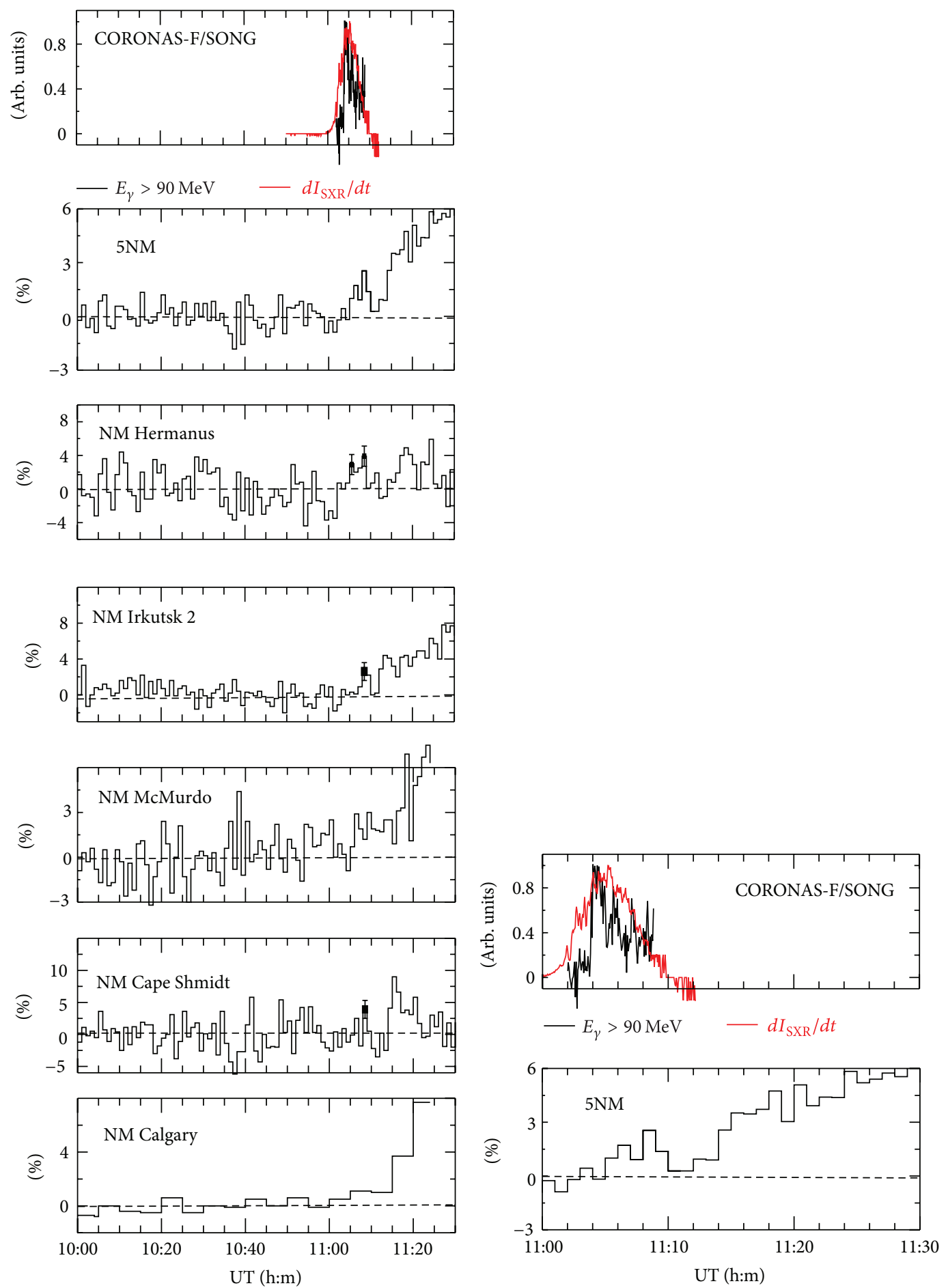

FiguRE 11: GLE 65, October 28, 2003. The left part, upper panel: SONG gamma-rays (black curve) and the soft X-ray derivative (red curve). The second panel: count rates of five NMs obtained by the method of superposed epoch. Five panels below: data of each NM separately. Right part: two upper panels from the left part displayed with better time resolution.

100-200 MeV when the impulsive phase of 4 powerful flares, namely, August 25, 2001, October 28, 2003, November 4, 2003, and January 20, 2005, was in progress. All these flares produced neutrons and/or protons, recorded near the Earth. We demonstrated that the SONG response was consistent with detection of the pion-decay emission and high-energy neutron fluxes.

High-energy neutrons and pion-decay photons are produced in the same interactions of accelerated protons with ambient matter instantly. So it would be reasonable to expect 

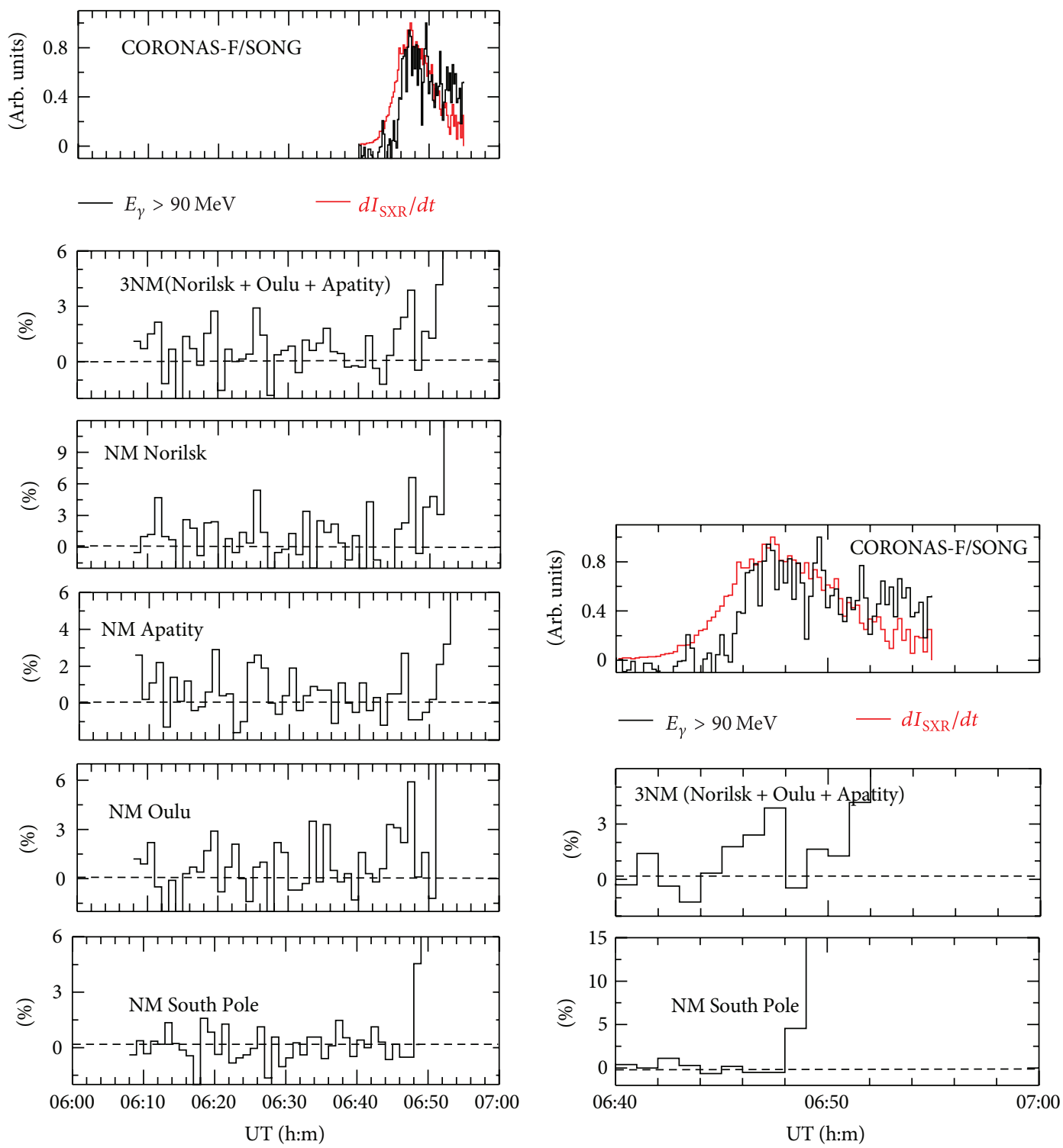

FIGURE 12: GLE 69, January 20, 2005. Left part, upper panel: SONG gamma-rays (black curve) and the soft X-ray derivative (red curve). The second panel: the count rates of three NMs obtained by the method of superposed epoch. Three panels below: Oulu, Apatity, and Norilsk NMs. The bottom panel: NM South Pole. Right: zoom of photon emissions and of count rates of 3NMs and NM South Pole.

TABLE 1: Timing of pion-decay gamma emission and high-energy particle onsets.

\begin{tabular}{lcccc}
\hline Date & August 25, 2001 & October 28, 2003 & November 4, 2003 & January 20, 2005 \\
\hline Flare importance, location & X5.3/3B & X17.2/4B & X $>28 / 4 \mathrm{~B}$ & X7.1/3B \\
& S17E34 & S16E08 & N19W83 & From 06:44:40 \\
Time of the pion-decay emission & $16: 31: 10-16: 32: 30$ & From 11:04:50 & $19: 42: 30-19: 48: 00$ & 19:45-19:55 \\
Time of proton onset & No & $11: 06-11: 08$ & $19: 48: 00-19: 48: 30$ & No \\
Time of neutron onset & $16: 35-16: 36$ & $11: 07: 00-11: 07: 30$ & \\
\hline
\end{tabular}

the observation of both in one and the same event. Indeed, neutrons were observed by SONG and NMs after three flares. On January 20, 2005, neither ground-level detectors nor CORONAS-F/SONG observed neutrons though the observation conditions were favorable for their detection by SONG. It is difficult to understand the absence of neutrons, since GLE 69 accompanying this flare reached $5400 \%$.

One can use any model of the incident photon spectrum to restore it from the detector pulse spectrum. In our simulation methods we performed the usual procedure of spectrum 

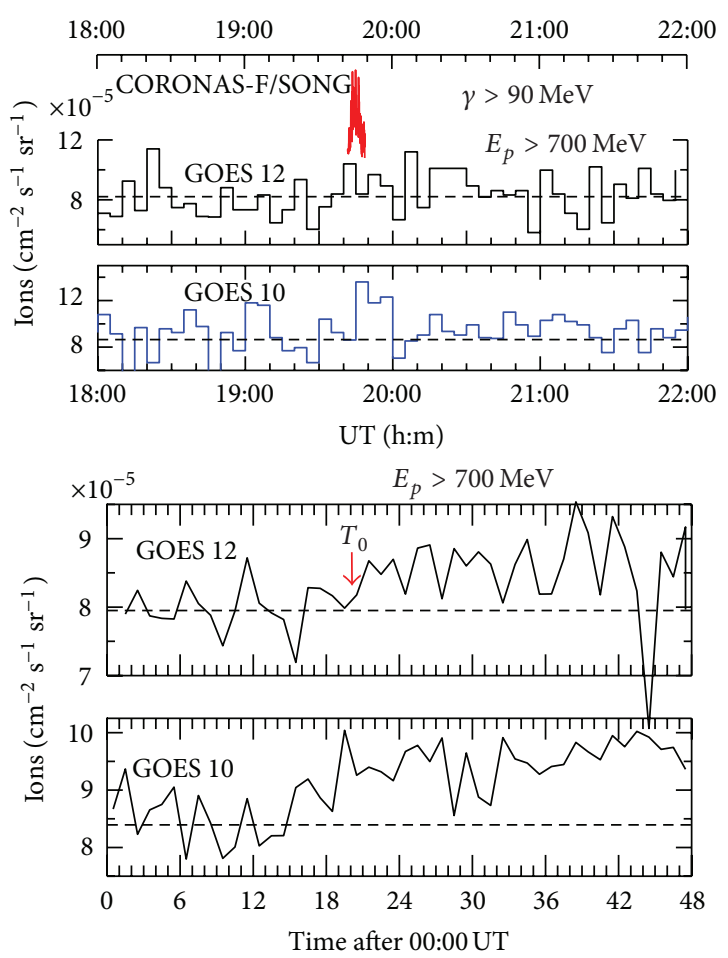

FIGURE 13: Time profiles of high-energy proton channels recorded by GOES-10 and -12 on November 4, 2003. Upper two panels: proton time profiles over 4 hours around the solar flare. Red curve in upper panel shows the increase observed in gamma-ray channel $>90 \mathrm{MeV}$. Two lower panels: proton time profiles measured by HEPAD during enlarged time period. $T_{0}=19: 42: 30$.

restoration, accepted by the scientific community studying high-energy gamma emission (see, e.g., $[16,22-24]$ ). The fitting procedure including standard $\chi^{2}$ permits to calculate the total incident spectrum and the pion-decay contribution. To elicit a pion-decay feature in the gamma-ray emission spectrum the intensity of the pion-decay emission must exceed the bremsstrahlung background. SONG spectrometer had the appropriate sensitivity and energy resolution for these purposes.

Exploring four flares we distinguished several acceleration episodes with substantially different characteristics during the time evolution of these flares which implies a substantial change in the spectrum shape of accelerated electrons as well as ions. We define maximum energy release as maximum $d I_{\text {SXR }} / d t$ in this work. It is known that at the beginning of the flare impulsive phase the value of $d I_{\text {SXR }} / d t$ does not exceed $20-30 \%$ of its peak value. Electrons are mainly accelerated during this time, which is manifested by bremsstrahlung hard X-ray and gamma-ray emission. The photon energy of such "pure electron spectrum" can reach 60-100 MeV. This was demonstrated earlier in June 21, 1980, May 24, 1990, and March 26, 1991, powerful flares [16, 21, 62].

The measurements of the narrow gamma-lines performed by SPI during October 28 and November 4, 2003, as well as RHESSI during January 20, 2005, events indicated the presence of protons accelerated up to $10-30 \mathrm{MeV}$ near the beginning of these events. We discovered that in the rise phase of August 25, 2001, and October 28, 2003, flares the energy of photons reached at least $80-100 \mathrm{MeV}$ without any deposit from the pion-decay emission. At the beginning of November 4, 2003, and January 20, 2005, flares the spectral feature associated with the pion-decay process was very faint. It means that the number of ions accelerated up to the energy above the threshold of pion production $(\sim 300 \mathrm{MeV})$ was very small in these time intervals.

We emphasize that observation of the pion-decay gamma emission is the method of investigating proton acceleration up to hundreds of $\mathrm{MeV}$ and more because this spectral feature cannot be imitated by other processes.

We determined the time of the sharp increase of the pion-decay component intensity. Since pion-decay gammarays are emitted by accelerated ions almost instantaneously, the moment of the sharp rise of the pion-decay component indicates the time of the proton bulk appearance in the low corona. Neutron generation function (or more precisely the injection function) can be determined by solving the inverse problem (e.g., $[49,63])$. The time of neutron production can be determined by this method with accuracy $\geq 1 \mathrm{~min}$; the accuracy derived from the pion-decay emission data is higher.

We found that the time of the maximum efficiency of proton acceleration is close to the time interval when the $d I_{\text {SXR }} / d t$ value exceeds 0.9 of its maximum. If our definition of flare energy release is correct, then this time closeness indicates that maximum efficiency of ion acceleration occurs in the time of flare energy release. The strong correlation between pion-decay flux time profile and magnetic-flux change rate was observed in October 28, 2003, flare (see Figure 5). Certainly it is only one case, but it implies that acceleration of high-energy protons may be an intrinsic property of the impulsive energy-release process, and it seems to be important for particle acceleration models.

We clearly saw that the increase in the electron energy (hardening of the spectral index) is not necessarily consistent with the episodes of the maximum efficiency of proton acceleration as it is clearly seen in October 28, 2003, flare.

Comparing the timing of pion-decay emission with the arrival time of the first energetic particles at $1 \mathrm{AU}$ we found that these particles lagged $\approx 3-5$ min behind the gamma-rays emission. So we proved that these protons were accelerated in the flare simultaneously with the interacted protons (or they both belong to the same population of accelerated particles).

Our investigation of three events revealed that the maximum pion production indicating the maximum efficiency of proton acceleration was observed within the time interval of maximum energy release determined by $d I_{\mathrm{SXR}} / d t$. This conclusion can be extended to the flare on November 4, 2003, if we assume that maximum energy release was determined from data on hard X-rays.

It is well known that for most of GLEs measurements of high-energy gamma emission from associated flares were absent. We showed in the present paper that the time of the most efficient proton acceleration coincided with the time interval of maximum energy release manifested by $d I_{\mathrm{SXR}} / d t$. Taking this result as the "null" hypothesis, we have used it as a working tool for determining the time delay of the first 
particles of GLE relative to the time of maximum efficiency of proton acceleration. We carried out a detailed study of the onset time of 43 GLEs and found that this delay did not exceed 10 minutes in 28 events [64]. This means that proton acceleration to relativistic energy starts typically within the time interval of maximum flare energy release.

The indirect methods of the initial moment determination and time duration of heavy particle acceleration up to relativistic energies (i.e., the analysis of radio emission, the determination of CME lift-off time, and the time of type II onset that are usually considered to be an indication of a shock wave in the corona, etc.) do not provide such a time accuracy.

\section{Conclusions}

We have analyzed high-energy gamma emission and neutron flux measurements of four solar flares: August 25, 2001, October 28, 2003, November 4, 2003, and January 20, 2005, obtained by CORONAS-F and combined with other observations of these flares. We showed that the SONG instrument response had been consistent with the detection of the piondecay gamma emission and neutron fluxes in these events. We determined the onset time of GLEs 65 and 69 which accompanied October 28, 2003, and January 20, 2005, flares on the basis of the Neutron Monitor World Network data. For reliable determination of this time we used the superposed epoch method. We used data of HEPAD detectors onboard GOES 10 and 12 to determine the onset time of high-energy protons during SPE on November 4, 2003. We obtained the following results.

(1) Observations of both pion-decay gamma-rays and high-energy neutrons in one and the same event prove undoubtedly that protons were accelerated up to relativistic energy, and then they interacted with solar matter. Detection of high-energy neutrons in the considered events proves that the observed feature in the gamma-ray spectrum was caused by the piondecay process. Acceleration of protons responsible for GLE/SPE serves as an additional argument to ascribe this feature to the pion decay.

(2) The pion-decay emission was observed within the time interval of the maximum flare energy release, which was manifested particularly by $d I_{\mathrm{SXR}} / d t$ time evolution. Hence, proton acceleration begins in the course of the flare but not later. However, we recognize that this conclusion is not the general rule. There are flares in which the pion-decay emission appeared only after the end of the main energy release.

(3) GLEs/SPE onset was observed with a short $<5 \mathrm{~min}$ delay in respect to the time of the pion-decay gammarays' observations, which is the least possible value. So, we suggest that in the events which took place on October 28, 2003, November 4, 2003, and January 20, 2005, both protons interacted in the solar atmosphere, and the first protons which arrived to the Earth belonged to one and the same population of the accelerated particles.
Revealed relationship between high-energy gamma-rays and GLE may serve as an additional element for short-time forecasts of radiation hazard storms in addition to those based on ground-based devices (NMs) and lower energies emission measurements. Thus, it makes sense to continue to provide regular patrol of high-energy "neutral emissions" from the Sun on various satellites and spacecraft in order to investigate space weather.

\section{Acknowledgments}

The authors are deeply indebted to G. Share and A.V. Belov for helpful discussions, R. Murphy for providing calculated pion-decay spectra, A. V. Belov for providing NM data set, V. V. Grechnev for providing Yohkoh data, and L. Kashapova for restoring the Yohkoh/HXS spectrum. This work was supported in part by the Russian Foundation for Basic Research (projects no. 05-02-17487 and 09-02-01145). $\mathrm{K}$. Kudela wishes to acknowledge VEGA agency (project 2/0040/13) for support.

\section{References}

[1] S. E. Forbush, "Three unusual cosmic-ray increases possibly due to charged particles from the sun," Physical Review, vol. 70, no. 9-10, pp. 771-772, 1946.

[2] A. V. Belov, E. A. Eroshenko, O. N. Kryakunova, V. G. Kurt, and V. G. Yanke, "Ground level enhancements of solar cosmic rays during the last three solar cycles," Geomagnetism and Aeronomy, vol. 50, no. 1, pp. 21-33, 2010.

[3] M. B. Kallenrode and G. Wibberenz, "Influence of interplanetary propagation on particle onsets," in Proceedings of the 21st International Cosmic Ray Conference, vol. 5, pp. 229-232, Adelaide, Australia, 1990.

[4] R. Ramaty and R. J. Murphy, "Nuclear processes and accelerated particles in solar flares," Space Science Reviews, vol. 45, no. 3-4, pp. 213-268, 1987.

[5] R. J. Murphy, C. D. Dermer, and R. Ramaty, "High-energy processes in solar flares," Astrophysical Journal Supplement Series, vol. 63, pp. 721-748, 1987.

[6] E. L. Chupp, D. J. Forrest, J. M. Ryan et al., "A direct observation of solar neutrons following the 01:18 UT flare on 1980 June 21," Astrophysical Journal Letters, vol. 263, pp. L95-L99, 1982.

[7] H. Debrunner, E. O. Flückiger, E. L. Chupp, and D. J. Forrest, "The solar cosmic ray neutron event on June 3, 1982," in Proceedings of the 18th International Cosmic Ray Conference, vol. 4, pp. 75-78, Bangalore, India, 1983.

[8] E. L. Chupp, H. Debrunner, E. O. Flückiger et al., "Solar neutron emissivity during the large flare on 1982 June 3," Astrophysical Journal, vol. 318, pp. 913-925, 1987.

[9] Y. E. Efimov, G. E. Kocharov, and K. Kudela, "On the solar neutrons observation on high mountain neutron monitor," in Proceedings of the 18th International Cosmic Ray Conference, vol. 10, pp. 276-278, Bangalore, India, 1983.

[10] P. Evenson, P. Meyer, and K. R. Pyle, "Protons from decay of solar flare neutrons," Astrophysical Journal, vol. 274, pp. 875882, 1983

[11] P. Evenson, R. Kroeger, P. Meyer, and D. Muller, "Solar flare neutron fluxes derived from interplanetary charged particle measurements," in Proceedings of the 18th International Cosmic Ray Conference, vol. 4, pp. 97-100, Bangalore, India, 1983. 
[12] Y. Muraki, "Solar neutrons and particle acceleration at the sun: what we learnt from solar neutron telescope," in Proceedings of the 30th International Cosmic Ray Conference, vol. 6, pp. 181-194, Merida, Mexico, 2009.

[13] R. J. Murphy, B. Kozlovsky, G. H. Share, X. M. Hua, and R. E. Lingenfelter, "Using gamma-ray and neutron emission to determine solar flare accelerated particle spectra and composition and the conditions within the flare magnetic loop," Astrophysical Journal Supplement Series, vol. 168, no. 1, pp. 167-194, 2007.

[14] Y. Hirasima, K. Okudaira, and T. Yamagami, "Solar gamma ray burst observed on 27 Sept. 1968," in Proceedings of the 11 International Cosmic Ray Conference, vol. 2, pp. 683-686, Budapest, Hungary, 1970.

[15] E. L. Chupp, D. J. Forrest, P. R. Higbie, A. N. Suri, C. Tsai, and P. P. Dunphy, "Solar gamma ray lines observed during the solar activity of August 2 to August 11, 1972," Nature, vol. 241, no. 5388, pp. 333-335, 1973.

[16] D. J. Forrest, W. T. Vestrand, E. L. Chupp et al., "Neutral pion production in solar flares," in Proceedings of the 19th International Cosmic Ray Conference, vol. 4, pp. 146-149, 1985.

[17] D. J. Forrest, W. T. Vestrand, E. L. Chupp, E. Rieger, J. Cooper, and G. H. Share, "Very energetic gamma-rays from the 3 June 1982 solar flare," Advances in Space Research, vol. 6, no. 6, pp. 115-118, 1986.

[18] P. P. Dunphy, E. L. Chupp, and E. Rieger, "Analysis of SMM GRS high-energy (>10 MeV) data from the solar flare of 1988 December 16," in Proceedings of the 21st International Cosmic Ray Conference, vol. 5, pp. 75-78, Adelaide, Australia, 1990.

[19] P. P. Dunphy and E. L. Chupp, "High-energy gamma-rays and neutrons from the solar flare of 1989 March 6," in Proceedings of the 22nd International Cosmic Ray Conference, vol. 3, pp. 65-68, Dublin, Ireland, 1991.

[20] O. V. Terekhov, R. A. Sunyaev, A. Y. Tkachenko et al., "Deuterium synthesis during the solar flare of March 22, 1991 (Granat data)," Astronomy Letters, vol. 22, no. 2, pp. 143-147, 1996.

[21] H. Debrunner, J. A. Lockwood, C. Barat et al., "Energetic neutrons, protons, and gamma rays during the 1990 May 24 solar cosmic-ray event," Astrophysical Journal, vol. 479, pp. 9971011, 1997.

[22] N. Vilmer, A. L. MacKinnon, G. Trottet, and C. Barat, "High energy particles accelerated during the large solar flare of 1990 May 24: X/ $\gamma$-ray observations," Astronomy and Astrophysics, vol. 412, pp. 865-874, 2003.

[23] E. J. Schneid, D. L. Bertsch, B. L. Dingus et al., "EGRET observations of X-class solar flares," Astronomy and Astrophysics Supplement Series, vol. 120, pp. 299-302, 1996.

[24] P. P. Dunphy, E. L. Chupp, D. L. Bertsch, E. J. Schneid, S. R. Gottesman, and G. Kanbach, "Gamma-rays and neutrons as a probe of flare proton spectra: the solar flare of 11 June 1991," Solar Physics, vol. 187, no. 1, pp. 45-57, 1999.

[25] V. V. Akimov, V. G. Afanassyev, A. S. Belousov et al., "Observation of high energy gamma-rays with the GAMMA-1 telescope (E>30 MeV)," in Proceedings of the 22nd International Cosmic Ray Conference, vol. 3, pp. 73-76, Dublin, Ireland, 1991.

[26] V. V. Akimov, P. Ambrož, A. V. Belov et al., "Evidence for prolonged acceleration based on a detailed analysis of the longduration solar gamma-ray flare of June 15, 1991," Solar Physics, vol. 166, no. 1, pp. 107-134, 1996.

[27] S. N. Kuznetsov, K. Kudela, I. N. Myagkova, A. N. Podorolsky, S. P. Ryumin, and B. Y. Yushkov, "First experience with SONG-M measurements on board CORONAS-F satellite," Indian Journal of Radio and Space Physics, vol. 33, no. 6, pp. 353-357, 2004.
[28] S. N. Kuznetsov, V. G. Kurt, I. N. Myagkova, B. Y. Yushkov, and K. Kudela, "Gamma-ray emission and neutrons from solar flares recorded by the SONG instrument in 2001-2004," Solar System Research, vol. 40, no. 2, pp. 104-110, 2006.

[29] V. G. Kurt, B. Y. Yushkov, K. Kudela, and V. I. Galkin, "Highenergy gamma radiation of solar flares as an indicator of acceleration of energetic protons," Cosmic Research, vol. 48, no. 1, pp. 70-79, 2010.

[30] E. L. Chupp, G. Trottet, P. P. Dunphy, and E. Rieger, "What we know and what we do not know about high energy neutral emissions from solar flares (a challenge for future missions)," in Proceedings of the 28th International Cosmic Ray Conference, pp. 3171-3174, Tsukuba, Japan, 2003.

[31] E. L. Chupp and J. M. Ryan, "High energy neutron and piondecay gamma-ray emissions from solar flares," Research in Astronomy and Astrophysics, vol. 9, no. 1, pp. 11-40, 2009.

[32] M. Ackermann, M. Ajello, A. Allafort et al., "FERMI detection of $\gamma$-ray emission from the M2 soft X-ray flare on 2010 June 12," Astrophysical Journal, vol. 745, pp. 144-154, 2012.

[33] N. Vilmer, A. L. MacKinnon, and G. J. Hurford, "Properties of energetic ions in the solar atmosphere from gamma-ray and neutron observations," Space Science Review, vol. 159, pp. 167224, 2011.

[34] L. I. Miroshnichenko and W. Q. Gan, "Particle acceleration and gamma rays in solar flares: recent observations and new modeling," Advances in Space Research, vol. 50, pp. 736-756, 2012.

[35] S. N. Kuznetsov, V. G. Kurt, B. Y. Yushkov, K. Kudela, and V. I. Galkin, "Gamma-ray and high-energy-neutron measurements on CORONAS-F during the solar flare of 28 October 2003," Solar Physics, vol. 268, no. 1, pp. 175-193, 2011.

[36] S. Masson, K. L. Klein, R. Bütikofer et al., "Acceleration of relativistic protons during the 20 January 2005 flare and CME," Solar Physics, vol. 257, pp. 305-322, 2009.

[37] I. A. Zhitnik, Y. I. Logachev, A. V. Bogomolov et al., "Polarization, temporal, and spectral parameters of solar flare hard $\mathrm{X}$-rays as measured by the SPR-N instrument onboard the CORONAS-F satellite," Solar System Research, vol. 40, no. 2, pp. 93-103, 2006.

[38] T. R. Metcalf, D. Alexander, H. S. Hudson, and D. W. Longcope, "Trace and Yohkoh observations of a white-light flare," Astrophysical Journal, vol. 595, pp. 483-492, 2003.

[39] M. Siarkowski and R. Falewicz, "Variations of the hard Xray footpoint asymmetry in a solar flare," Astronomy and Astrophysics, vol. 428, no. 1, pp. 219-226, 2004.

[40] J. P. Raulin, V. S. Makhmutov, P. Kaufmann et al., "Analysis of the impulsive phase of a solar flare at submillimeter wavelengths," Solar Physics, vol. 223, no. 1-2, pp. 181-199, 2004.

[41] V. S. Makhmutov, V. G. Kurt, B. Yu. Yushkov et al., "Spectral peculiarities of high energy X-ray radiation, gamma radiation, and submillimeter radio emission in the impulsive phase of a solar flare," Bulletin of the Russian Academy of Sciences, Physics, vol. 75, pp. 747-750, 2011.

[42] J. Sato, Y. Matsumoto, K. Yoshimura et al., "Yohkoh/WBS recalibration and a comprehensive catalogue of solar flares observed by Yohkoh SXT, HXT and WBS instruments," Solar Physics, vol. 236, no. 2, pp. 351-368, 2006.

[43] V. G. Kurt, S. I. Svertilov, B. Y. Yushkov et al., "Dynamics and energetics of the thermal and nonthermal components in the solar flare of January 20, 2005, based on data from hard electromagnetic radiation detectors onboard the CORONAS-F satellite," Astronomy Letters, vol. 36, no. 4, pp. 280-291, 2010. 
[44] K. Watanabe, Y. Muraki, Y. Matsubara et al., "Solar neutron event in association with a large solar flare on August 25, 2001," in Proceedings of the 28th International Cosmic Ray Conference, pp. 3179-3182, Tsukuba, Japan, 2003.

[45] S. N. Kuznetsov, V. G. Kurt, B. Y. Yushkov et al., "28 october 2003 flare: high-energy gamma emission, type II radio emission and solar particle observations," International Journal of Modern Physics A, vol. 20, no. 29, pp. 6705-6707, 2005.

[46] J. Kiener, M. Gros, V. Tatischeff, and G. Weidenspointner, "Properties of the energetic particle distributions during the October 28, 2003 solar flare from INTEGRAL/SPI observations," Astronomy and Astrophysics, vol. 445, pp. 725-733, 2006.

[47] G. Trottet, S. Krucker, T. Lüthi, and A. Magun, "Radio submillimeter and $\gamma$-ray observations of the 2003 October 28 solar flare," Astrophysical Journal, vol. 678, pp. 509-514, 2008.

[48] C. H. Miklenic, A. M. Veronig, and B. Vršnak, "Temporal comparison of nonthermal flare emission and magnetic-flux change rates," Astronomy and Astrophysics, vol. 499, pp. 893-904, 2009.

[49] J. W. Bieber, J. Clem, P. Evenson, R. Pyle, D. Ruffolo, and A. Sáiz, "Relativistic solar neutrons and protons on 28 October 2003," Geophysical Research Letters, vol. 32, no. 3, article L03S02, 5 pages, 2005.

[50] P. Kaufmann, J. P. Raulin, C. G. Giménez de Castro et al., "A new solar burst spectral component emitting only in the terahertz range," Astrophysical Journal Letters, vol. 603, pp. L121-L124, 2004.

[51] S. R. Kane, J. M. McTiernan, and K. Hurley, "Multispacecraft observations of the hard X-ray emission from the giant solar flare on 2003 November 4," Astronomy and Astrophysics, vol. 433, no. 3, pp. 1133-1138, 2005.

[52] P. Kaufmann, G. D. Holman, Y. Su et al., "Unusual emissions at various energies prior to the impulsive phase of the large solar flare and coronal mass ejection of 4 November 2003," Solar Physics, vol. 279, pp. 465-475, 2012.

[53] K. Watanabe, M. Gros, P. H. Stoker et al., "Solar neutron events of 2003 October-november," Astrophysical Journal, vol. 636, pp. 1135-1144, 2006.

[54] V. V. Grechnev, V. G. Kurt, I. M. Chertok et al., "An extreme solar event of 20 January 2005: properties of the flare and the origin of energetic particles," Solar Physics, vol. 252, no. 1, pp. 149-177, 2008.

[55] C. Bouratzis, P. Preka-Papadema, A. Hillaris et al., "Radio observations of the 20 January $2005 \mathrm{X}$-class Flare," Solar Physics, vol. 267, no. 2, pp. 343-359, 2010.

[56] V. V. Zharkova, N. S. Meshalkina, L. K. Kashapova, A. T. Altyntsev, and A. A. Kuznetsov, "Effect of a self-induced electric field on the electron beam kinetics and resulting hard X-ray and microwave emissions in flares," Geomagnetism and Aeronomy, vol. 51, pp. 1029-1040, 2011.

[57] C. Pei, J. R. Jokipii, and J. Giacalone, "Effect of a random magnetic field on the onset times of solar particle events," Astrophysical Journal, vol. 641, pp. 1222-1226, 2006.

[58] K. L. Klein, S. Masson, R. Miteva, S. Samwel, O. Malandraki, and G. Trottet, "The Sun-Earth connection of energetic particles," EAS Publications Series, vol. 55, pp. 321-326, 2012.

[59] S. Masson, P. Démoulin, S. Dasso, and K. L. Klein, "The interplanetary magnetic structure that guides solar relativistic particles," Astronomy and Astrophysics, vol. 538, article A32, 14 pages, 2012.

[60] C. Plainaki, A. Belov, E. Eroshenko, H. Mavromichalaki, and V. Yanke, "Modeling ground level enhancements: event of 20
January 2005," Journal of Geophysical Research A, vol. 112, no. 4, article A04102, 16 pages, 2007.

[61] V. G. Kurt, B. Y. Yushkov, and A. V. Belov, "On the ground level enhancement beginning," Astronomy Letters, vol. 36, no. 7, pp. 520-530, 2010.

[62] V. V. Akimov, N. G. Leikov, V. G. Kurt, and I. M. Chertok, "The GAMMA-1 data on the March 26, 1991 solar flare," in Proceedings of AIP Conference. High-energy solar phenomenaa new era of spacecraft, vol. 294, pp. 106-111, 1994.

[63] A. V. Belov and M. A. Livshits, "Neutron burst on May 24, 1990," Astronomy Letters, vol. 21, pp. 37-40, 1995.

[64] V. Kurt, B. Yushkov, A. Belov, I. Chertok, and V. Grechnev, "Determination of acceleration time of protons responsible for the GLE onset," Journal of Physics: Conference Series, http://conferenceseries.iop.org/content/home. In press. 

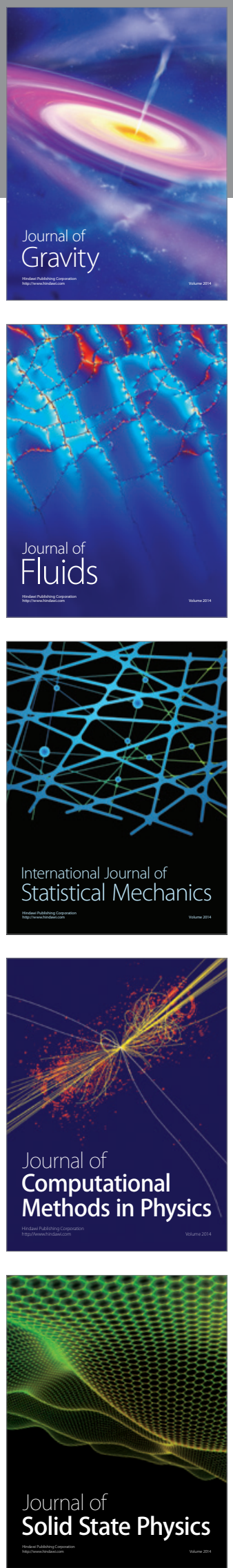

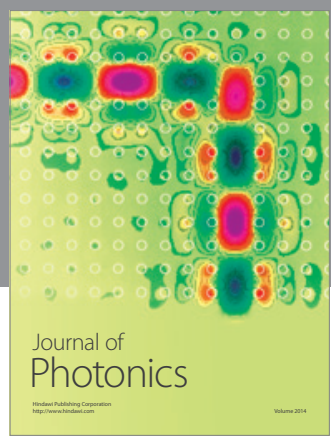

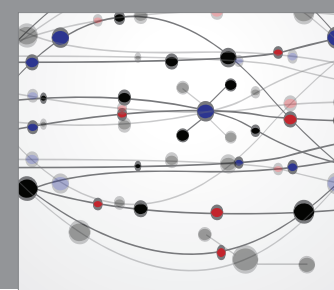

The Scientific World Journal

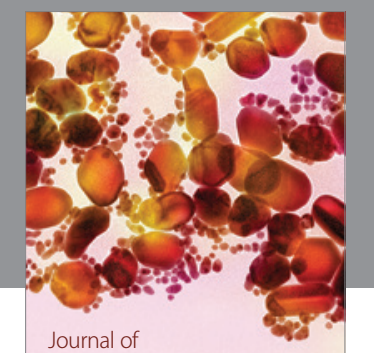

Soft Matter
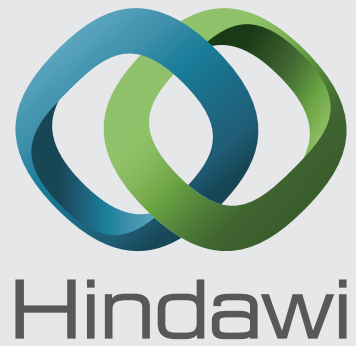

Submit your manuscripts at

http://www.hindawi.com
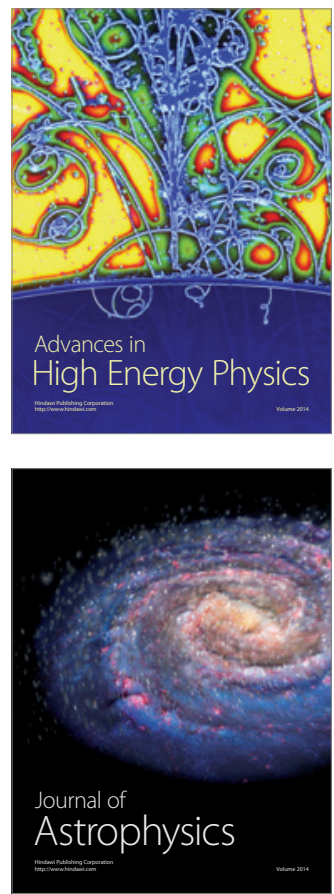
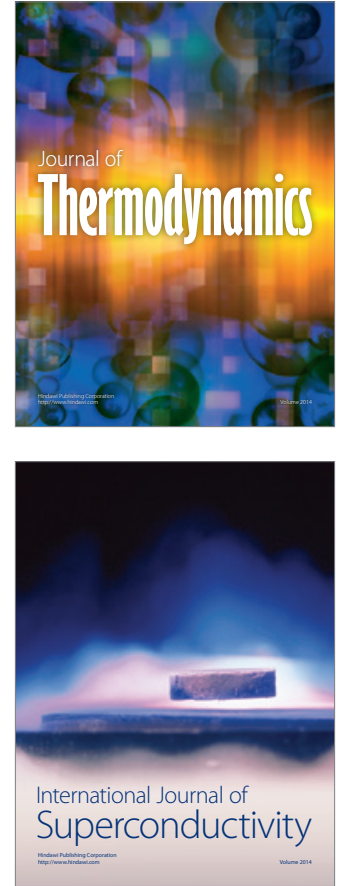
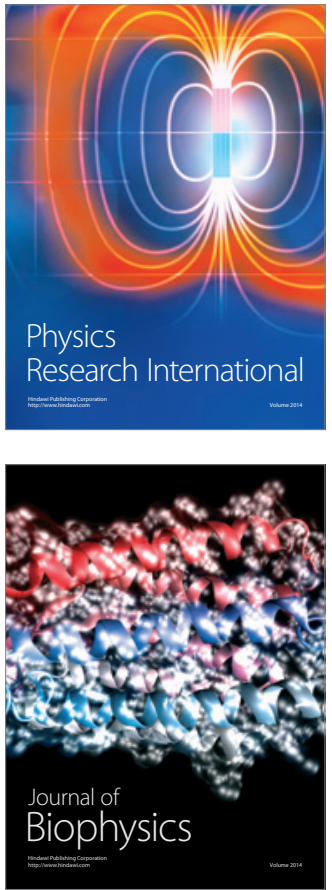
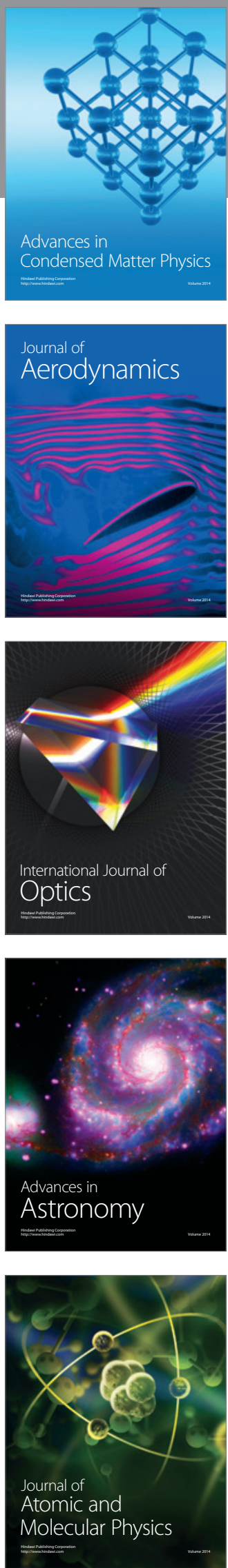